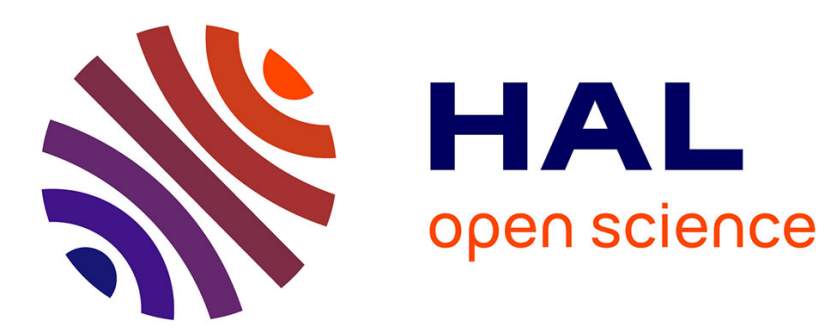

\title{
Using a High Order Sliding Modes for Diving Control a Torpedo Autonomous Underwater Vehicle
}

Tomas Salgado-Jimenez, Bruno Jouvencel

\section{To cite this version:}

Tomas Salgado-Jimenez, Bruno Jouvencel. Using a High Order Sliding Modes for Diving Control a Torpedo Autonomous Underwater Vehicle. OCEANS, Sep 2003, San Diego, CA, United States. pp.934-939, 10.1109/OCEANS.2003.178450 . lirmm-00269449

\section{HAL Id: lirmm-00269449 \\ https://hal-lirmm.ccsd.cnrs.fr/lirmm-00269449}

Submitted on 3 Apr 2008

HAL is a multi-disciplinary open access archive for the deposit and dissemination of scientific research documents, whether they are published or not. The documents may come from teaching and research institutions in France or abroad, or from public or private research centers.
L'archive ouverte pluridisciplinaire HAL, est destinée au dépôt et à la diffusion de documents scientifiques de niveau recherche, publiés ou non, émanant des établissements d'enseignement et de recherche français ou étrangers, des laboratoires publics ou privés. 


\title{
Using a High Order Sliding Modes for diving control a torpedo Autonomous Underwater Vehicle
}

\author{
T. Salgado-Jiménez ${ }^{1}$ and B. Jouvencel \\ LIRMM - University of Montpellier II \\ 161 rue Ada, 34392,Montpellier, Cedex 5, France \\ salgado@lirmm.fr, jouvencel@lirmm.fr
}

\begin{abstract}
In this paper, a High Order Sliding Modes Control (HOSMC) is used to control TAIPAN, a torpedo shaped AUV from LIRMM, France. The implementation of a Second Order Sliding Mode controller in this system is the main contribution of this work. Sliding Mode Control (SMC) is adequate for controlling AUVs, since it offers robustness in the presence of uncertainties parameters and environmental disturbances, however the main drawback is the chattering effects that stimulates high frequency vibration that can damage the actuators. HOSMC control preserves the properties of standard SMC and removes the chattering effects. The design of Proportional Derived (PD), SMC and HOSMC controller for control TAIPAN depth are described. A comparative study between the control laws is presented. The nonlinear hydrodynamic model of TAIPAN is used in the numerical simulations. Simulation results, that enlighten performance of the automatic controllers are showed.
\end{abstract}

\section{Introduction}

Controlling an Autonomous Underwater Vehicle AUV is not a trivial task, due to: Parameter uncertainties (as added mass, hydrodynamic coefficients, lift and drag forces), high and coupled non linearities and environmental disturbances (like ocean currents and wave's effects). Furthermore, the controller must satisfy two basis requirements: first it has to be enough sophisticated to develop his work; secondly, it shouldn't be very complicated, otherwise it could have singularities and their real time performance could be slow. Many control strategies have been adopted for AUV's depth and steering control: Optimal control [4], Neural Network [7], Adaptive Sliding Mode Control [3], etc . The most typical control laws used by the industry and research underwater robots are basically two: PD [2] [10] and Sliding Modes Control (SMC) [6] [11]. These algorithms are designed using a linear model of the robot.

Recently, a new control method called High Order Sliding Modes Control (HOSMC) was developed, and has been successfully applied in real time and simulation to several systems [1],[8]. Its principal characteristic is that it keeps the main advantages of the SMC removing the chattering effects.
In this paper, $\mathrm{PD}, \mathrm{SMC}$ and HOSMC control laws are tested in simulation, in order to maintain a desired depth. The implementation of a Second Order sliding mode control in TAIPAN is the main contribution of this work. TAIPAN is an AUV developed at LIRMM, France. This vehicle has a length of $1.9 \mathrm{~m}$, a maximum diameter of $0.25 \mathrm{~m}$ and a weight of $40 \mathrm{Kg}$. Figure (1) shows a picture of TAIPAN (for more details see [12]).

This document is organized as follows; TAIPAN general equation of motion and depth linear equation of motion are described in section II. AUV most typical control laws as PD and SMC are given in sections III and IV respectively. HOSMC is introduced in section $\mathrm{V}$. Finally, section VI presents the conclusion and future work.

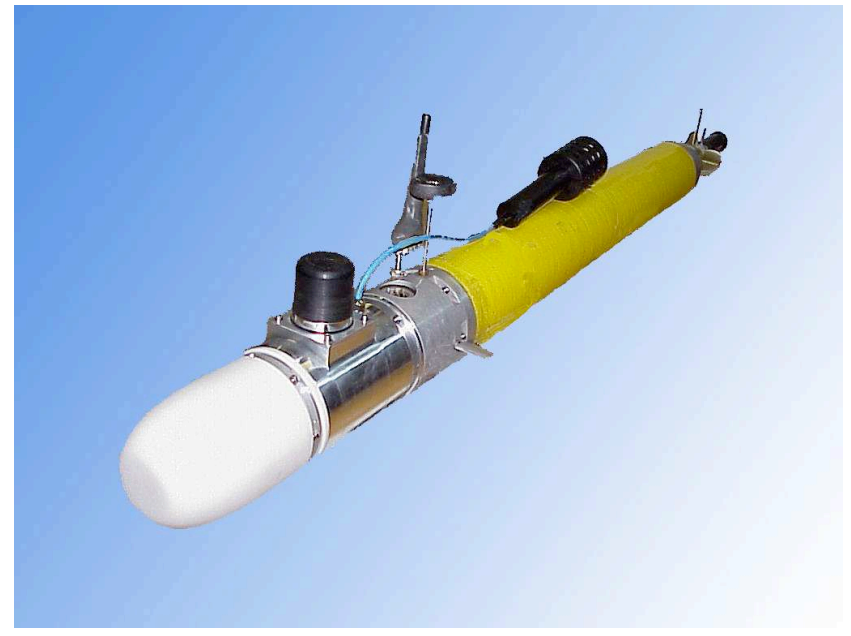

Fig. 1. The Autonomous Underwater Vehicle TAIPAN.

\section{TAIPAN Mathematical Model}

\section{A. Nonlinear general equation of motion}

The model used here, is according with Fossen [5]. He suggests that the general motion of an AUV can be described by using a Body-fixed frame relative to an Earthfixed frame (see Figure 2). The Body-fixed frame has components of motion given by:

Where

$$
v=\left[v_{1}, v_{2}\right]
$$

${ }^{1}$ Author thanks CONACyT, Mexico, for the financial support. 
$v_{1}=[u, v, w]$ Linear velocities

$v_{2}=[p, q, r]$ Angular velocities

The position of the Body-fixed measured in the Earthfixed is,

$$
\eta=\left[\eta_{1}, \eta_{2}\right]
$$

where

$\eta_{1}=[x, y, z]$ position of the origin

$\eta_{2}=[\phi, \theta, \psi]$ orientation angles of roll $(\phi)$, pitch $(\theta)$ and yaw $(\psi)$.

The non linear vehicle equation of motion, represented in the Body-fixed frame is written as [5]:

$$
\begin{gathered}
M \dot{v}+c(v) v+D c(v) v+g(\eta)=\tau \\
\dot{\eta}=J(\eta) v
\end{gathered}
$$

Where

$M=M_{r b}+M_{a}$ is the inertia and added mass matrix.

$C=C_{r b}+C_{a}$ is the matrix of Coriolis and centripetal forces, from inertia and hydrodynamics.

$D$ is the hydrodynamic damping.

$g$ is the vector of restoring forces and moments.

$\dot{\eta}=J(\eta) v \quad$ is the transformation matrix between the Body and Earth frames.

Control inputs vector $\tau$ has three components:

$$
\tau=\left[\delta_{r}, \delta_{S}, n\right]
$$

where $\delta_{s}$ is the surface deflection from rudder, $\delta_{r}$ is the stern deflection and $n$ propeller revolutions.

The buoyancy adjustment is zero because TAIPAN is approximately neutrally buoyant.

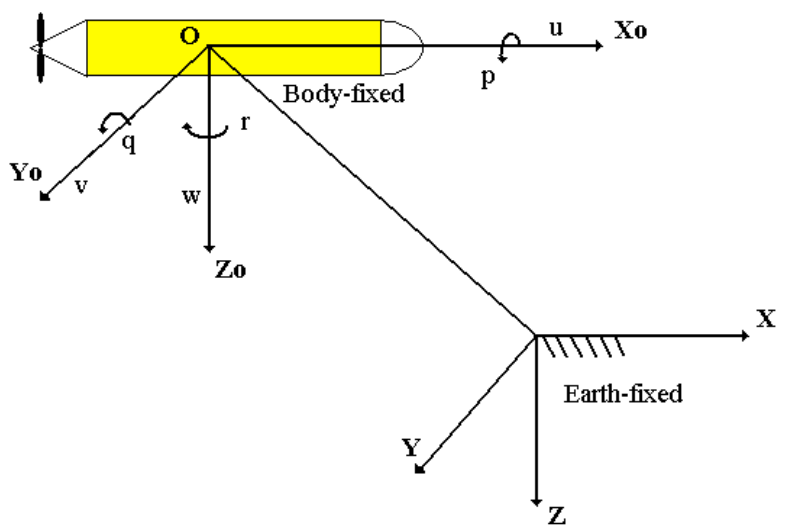

Fig. 2. TAIPAN Body-fixed and Earth-fixed frames.

\section{B. Depth Plane linear equation of motion}

The depth plane kinematics model is:

$$
\begin{aligned}
& \dot{x}=\cos (\theta) u+\sin (\theta) w \\
& \dot{z}=-\sin (\theta) u+\cos (\theta) w \\
& \dot{\theta}=q
\end{aligned}
$$

where $u$ and $w$ are the forward and heave velocities, of the vehicle.

Equation (2.1) can beinearized around a steady point. Applying the Taylor series expression, the following kinematics relationship is obtained:

$$
\begin{aligned}
& \dot{x}=u+\theta w \\
& \dot{z}=-\theta u_{0}+w \\
& \dot{\theta}=q
\end{aligned}
$$

In order to determinate a depth plane dynamics equation of motion, all unrelated terms $\left(v, p, r, y_{g}\right.$ and $\left.x_{g}\right)$ will be set to zero. Using the Newton laws, the following simplified system is obtained:

$$
\begin{aligned}
& m\left[\dot{u}+z_{g} \dot{q}\right]=\Sigma X \\
& m\left[\dot{w}+x_{g} \dot{q}-u_{0} q\right]=\Sigma Z \\
& I_{y} q+m\left[\dot{u} z_{g}+x_{g}\left(w-u_{0} q\right)\right]=\Sigma M
\end{aligned}
$$

The external forces $X, Y, Z$ are described by;

$$
\begin{aligned}
& \Sigma X=X_{\dot{u}} \dot{u}+X_{u} u+X_{q} q+X_{\theta} \theta \\
& \Sigma Z=Z_{\dot{w}} \dot{w}+Z_{\dot{q}} \dot{q}+Z_{w} w+Z_{q} q+Z_{\delta_{S}} \delta_{S} \\
& \Sigma M=M_{\dot{w}} \dot{w}+M_{\dot{q}} \dot{q}+M_{w} w+M_{q} q+M_{\theta} \theta+M_{\delta_{S}} \delta_{S}
\end{aligned}
$$

The expression (2.4) is composed by linear terms of hydrostatic, stern deflection, added mass and linear damping. It is known that the speed $u$ is constant and $z_{\mathrm{g}}$ is small compared to the other terms, so it's possible to decouple surge $(x)$ from the system. From (2.3), (2.4) and (2.2) linear depth equation is obtained:

$$
\left[\begin{array}{cccc}
m-z_{\dot{W}} & -z_{\dot{q}} & 0 & 0 \\
-M_{\dot{w}} & I_{y}-M_{\dot{w}} & 0 & 0 \\
0 & 0 & 1 & 0 \\
0 & 0 & 0 & 1
\end{array}\right]\left[\begin{array}{c}
\dot{w} \\
\dot{q} \\
\dot{\theta} \\
\dot{z}
\end{array}\right]+\left[\begin{array}{cccc}
-z_{w} & m-u_{0} Z_{q} & 0 & 0 \\
-M_{w} & -M_{q} & M_{\theta} & 0 \\
0 & -1 & 0 & 0 \\
-1 & 0 & u_{0} & 0
\end{array}\right]\left[\begin{array}{c}
w \\
q \\
\theta \\
z
\end{array}\right]=\left[\begin{array}{c}
Z_{\delta} \\
M_{\delta} \\
0 \\
0
\end{array}\right] \delta_{S}
$$

Equation (2.5) can be written as the typical representation of a linear systems:

$$
\dot{x}=A x+B u
$$

where $x=[w, q, \theta, z]^{T}$ and $u=\delta_{s}$.

In experimental simulations a constant forward speed $u_{0}=1 \mathrm{~m} / \mathrm{seg}$ is used, noise and perturbation are not considered. Initial depth is 0 meters and the control objective is to reach 2 meters. 


\section{Proportional Derivative Control (PD)}

In this section a PD controller is designed and implemented in TAIPAN in order to reach a desired depth. Assuming that the heave velocity $w$ during diving is small compared to the other terms, the linear model (2.5) is reduced into the following system:

$$
\left[\begin{array}{c}
\dot{q} \\
\dot{\theta} \\
\dot{z}
\end{array}\right]=\left[\begin{array}{ccc}
\frac{M_{q}}{I_{y}-M_{\dot{q}}} & \frac{M_{\theta}}{I_{y}-M_{\dot{q}}} & 0 \\
1 & 0 & 0 \\
0 & -u_{0} & 0
\end{array}\right]\left[\begin{array}{c}
q \\
\theta \\
z
\end{array}\right]+\left[\begin{array}{c}
\frac{M_{\delta}}{I_{y}-M_{\dot{q}}} \\
0 \\
0 \\
\end{array}\right] \delta_{S}
$$

Applying thaplace transformation to this reduced model, pitch and depth transfer functions are obtained:

Pitch transfer function; which relates input stern angles $\delta_{\mathrm{s}}$ to the output vehicle pitch $\theta$ :

$$
\frac{\theta(s)}{\delta_{s}(s)}=\frac{\frac{M_{\delta_{s}}}{I_{y}-M_{\dot{q}}}}{s^{2}-\frac{M_{q}}{I_{y}-M_{\dot{q}}} S-\frac{M_{\theta}}{I_{y}-M_{\dot{q}}}}
$$

Depth transfer function; relates the input pitch angle $\theta$ to the depth $z$ :

$$
\frac{z(s)}{\theta(s)}=-\frac{u_{0}}{s}
$$

In order to control TAIPAN, a controller proposed by Bjorn [2] is used. This controller is composed by a PD control for pitch, and a proportional control for depth. The control law is expressed by:

$$
\delta_{S}=-G_{1}\left(z-z_{d}\right)+G_{2} \theta+G_{3} q
$$

The $G_{2}$ et $G_{3}$ are designed to have a desired closed-loop dynamics. As a desired system the next poles are chosen,

$$
S_{1,2}=-3 \pm j 2 .
$$

$G_{l}$ has to be chosen to guarantee that the pitch loop response is faster than the depth loop response. The calculated gains are $G_{l}=2.0, G_{2}=5.209, G_{3}=12.963$. Figure (3) shows the results of this law in TAIPAN. The depth response to $\mathrm{PD}$ law is exponential and slow. $q$ et $\theta$ present oscillations due to model nonlinearities. This controller will be improved by the addition of Integral part.

\section{Sliding Modes Control (SMC)}

In this section a SMC is used to control theTAIPAN's depth. This technique is normally used when the condition is to have a robust control under parameters uncertainties and unknown perturbations. The design methodology is based in [6]. Sliding model control is composed by two parts:

$\tau=\hat{\tau}+\bar{\tau}$

$\hat{\tau}$ Nominal control, which is determined by the robot model (in this case the linearized dynamic model (2.5)).

$\bar{\tau} \quad$ Sliding part, which is useful to compensate model uncertainties.

In order to design a SMC, we must define a sliding surface in the state space error, guaranteeing for this way a global stability of the system. The error state space is:

$$
\tilde{x}=\left[w-w_{d}, q-q_{d}, \theta-\theta_{d}, z-z_{d}\right]
$$

Using $\tilde{x}$ the sliding surface is built:

$$
\sigma=\left[S_{1}, S_{2}, S_{3}, S_{4}-\left[\begin{array}{c}
\tilde{\mathcal{w}} \\
\tilde{q} \\
\widetilde{z}
\end{array}\right]=S^{T} x\right.
$$

where $S_{1}, S_{2}, S_{3}, S_{4}$ are the sliding surface constants that will be calculated later. The sliding surface $\sigma$ must obey the next condition:

$$
\dot{\sigma} \rightarrow 0 \text { and } \sigma \rightarrow 0 \text { as } t \rightarrow \infty
$$

so, this imply $\tilde{x} \rightarrow 0$ as $t \rightarrow \infty$.

Previous conditions are guaranteed with the following Lyapunov function:

$$
V(\sigma)=0.5(\sigma)^{2}
$$

The condition $\sigma=0$ is reached in a finite time if:

$$
\dot{\sigma}=-\eta \operatorname{sgn}(\sigma)
$$

Deriving (4.1), we get $\dot{\sigma}=S^{T} \dot{x}$. Replacing (2.6) in the previous expression:

$$
S^{T}=(A x+B u)=-\eta \operatorname{sgn}(\sigma)
$$

Then, the control inputs are:

$\tau=-\left(S^{T} B\right)^{-1} S^{T} A x-\eta^{2}\left(S^{T} B\right) \operatorname{sgn}(\sigma)$

$\tau=\hat{\tau}+\bar{\tau}$

The two components of the control are:

$$
\hat{\boldsymbol{\tau}}=-\left(S^{T} B\right)^{-1} S^{T} A x
$$

and

$$
\bar{\tau}=-\eta^{2}\left(S^{T} B\right) \operatorname{sgn}(\sigma)
$$

The feedback law (4.2) is designed so that the system 
has a desired behavior, when it is in the sliding plane (in other words $\sigma=0$ ). Substituting (4.2) into (2.6), we get the closed - loop dynamics:

or

$$
\dot{x}=\left[A-\left(S^{T} B\right)^{-1} S^{T} A\right] x
$$

$$
\begin{gathered}
\dot{x}=\lfloor A-B k\rfloor x \\
A_{C}=A-B k
\end{gathered}
$$

where $k$ is a gain vector calculated by a pole placement method. The closed - loop poles of the system are, $\lambda_{1}=0$, and $\lambda_{i} i=2, \ldots, n$.

The sliding surface coefficients $S$ are elements of the left eigenvector of the closed - loop dynamics matrices $A_{c}$, corresponding to a pole at the origin.

$$
S^{T}=\left\lfloor A_{C}\right\rfloor=0
$$

For TAIPAN, we choose the desired poles $[0,-0.5,-0.6$, $-0.7]$ and the vector gain $k$ calculated is, $k=[-4.905$, $1.9838,-0.5528$, 0]. using (4.4), $S=[-1.0,-2.4572,-2.2206$, $0.7364]$.

Finally the sliding surface and the control output are:

$\sigma=\left[1\left(w-w_{d}\right)-2.4572\left(q-q_{d}\right)-2.2206\left(\theta-\theta_{d}\right)+0.7364\left(z-z_{d}\right)\right]$

$\delta_{s}=[-4.905 w-1.9838 q+0.5528 \theta+2.5 \tanh (\sigma / 0.4)]$

The discontinuous functionsgn in (4.5) is replaced by a continuous function tanh. This change allows us to eliminate chattering induced by the $s g n$ function. The AUV's answer is showed in Figure (4). $z, \theta$, and $q$ have an asymptotical convergence to its desires values, SMC is faster than the PD controller.

\section{High Order Sliding Modes Control (HOSMC)}

In the standard SMC $\dot{\sigma}$, is discontinuous; this is the main reason why high frequency switching appears in the output signal (chattering effect), which causes problems in practical application. In order to avoid chattering, in this section a high order sliding control is used [8][9]. HOSMC acts on the higher order time derivative of the system deviation, instead of influencing the first deviation derivative as it happens in SMC [9]. Its principal characteristic is that it keeps the main advantages of the SMC, removing the chattering effects. The sliding order is a number of continuous total derivatives of $\sigma$ in the vicinity of the sliding mode. It fix the dynamics smoothness degree. The $r$-th order sliding mode ( $r$-sliding) is determined by the equation:

$$
\sigma=\dot{\sigma}=\ddot{\sigma}=\cdots=\sigma^{(r-1)}
$$

The Relative Degree (RD); is the continuous derivatives of $\sigma$ defined as [9]:

$$
\begin{aligned}
& \text { 1) } \mathrm{RD} \quad r=1 \text {, i.e., } \frac{\partial}{\partial u} \dot{\sigma} 0 \\
& \text { 2) } \mathrm{RD} \quad r=2 \text {, i.e., } \frac{\partial}{\partial u} \sigma^{(i)}=0(i=1,2, \ldots, r-1), \frac{\partial}{\partial u} \sigma^{(r)} \quad 0
\end{aligned}
$$

The main problem of HOSMC is the increment of the demanded information. For example, $r=3$, a 3-sliding controller needs as an input control $\sigma=\dot{\sigma}=\ddot{\sigma}$. The exclusion of this disadvantage are the 2-sliding 'Twisting' and 'Super Twisting' algorithms, that needs only the measure of $\sigma$. This two techniques are used here.

\section{A. 2-sliding controllers for TAIPAN}

The sliding surface (4.1), is a smooth function which should be keep at zero by the control $(\sigma=0)$. Indeed, it is successively derived like in (5.1), by depending on the relative degree of the system. For this case, the relative degree of the system is 1 , this means that control appears in the first total time derivative of $\sigma$. So the control condition for our case is $\sigma=\dot{\sigma}=0$. According to [8] and [9] the 2-sliding algorithm for a system with a relative degree is 1 :

\section{A.1. Twisting}

It's called Twisting because its trajectory follows an infinite number of rotations, while converges to zero.

$$
\dot{u}=\left\{\begin{array}{clc}
-u & \text { if } & |u|>1 \\
-V_{m} \operatorname{sgn}(\sigma) & \text { if } & \sigma \dot{\sigma} \leq 0 ;|u| \leq 1 \\
-V_{M} \operatorname{sgn}(\sigma) & \text { if } & \sigma \dot{\sigma}>0 ;|u| \leq 1
\end{array}\right.
$$

The condition for finite time convergence to the sliding manifold $\sigma$ are [8][9]:

$$
V_{M}>V_{m}, \quad V_{m}>\frac{4 \Gamma_{M}}{\sigma_{0}}, \quad V_{m}>\frac{\Phi}{\Gamma_{m}} \text { and } \quad \Gamma_{m} V_{m}-\Phi>\Gamma_{M} V_{m}-\Phi
$$

\section{A.2. Super Twisting}

This controller was developed to control systems with relative degree 1 . It is composed by two parts. The first is defined in terms of a discontinuous time derivative $u_{l}$, while the second is defined by the continuous function of the sliding variable $u_{2}$ :

$$
\begin{aligned}
& u=u_{1}+u_{2} \\
& \dot{u}_{1}=\left\{\begin{array}{c}
-u \\
-W \operatorname{sgn}(\sigma) \text { if }|u|>1
\end{array}\right.
\end{aligned}
$$


$u_{2}=\left\{\begin{array}{l}-\lambda\left|\sigma_{0}\right|^{\rho} \operatorname{sgn}(\sigma) \text { if }|\sigma|>\sigma_{0} \\ -\lambda|\sigma|^{\rho} \operatorname{sgn}(\sigma) \text { if }|\sigma| \leq \sigma_{0}\end{array}\right.$

In this case, the finite time convergence conditions are:

$W>\frac{\Phi}{\Gamma_{M}}, \quad 0<\rho \leq 0.5$ and $\lambda^{2} \geq \frac{4 \Phi \Gamma_{M}(W+\Phi)}{\Gamma_{m}^{2} \Gamma_{m}(W-\Phi)}$

Twisting and Super Twisting algorithms have advantages and disadvantages. They don't need any information on the time derivative of the sliding variable $(\sigma)$, reducing in this way the computational burden of the controller. Super Twisting is very robust, but the control output is not Lipschitzian with small $\sigma$, which may cause some noise in control output. Twisting produces a Lipschitzian control signal, but its convergence is slower [8]. It is possible to see this convergence speed in the Figures (5) and (6).

In practice the most convenient way to find appropriate values in (5.2) and (5.3) is to adjust them during computer simulation [8]. The control constants used in our simulations are:

Twisting

$$
V m=0.25 \text { and } V M=0.8
$$

Super Twisting

$$
W=0.4, \lambda=1.0, \rho=0.5 \text { and } \sigma_{0}=0.05
$$

The sliding surface $(\sigma)$ and the nominal control $(\hat{\boldsymbol{\tau}})$ used here, were calculated in section IV:

$$
\begin{aligned}
\sigma & =\left[1\left(w-w_{d}\right)-2.4572\left(q-q_{d}\right)-2.2206\left(\theta-\theta_{d}\right)+0.7364\left(z-z_{d}\right)\right] \\
\hat{\boldsymbol{\tau}} & =(-4.905 w-1.9838 q+0.5528 \theta)
\end{aligned}
$$

Finally, the HOSMC output is expressed by the following equation:

$$
\delta_{S}=\hat{\tau}+u
$$

Whene is the high order control component and is defined by the equation (5.2) for the Twisting, and (5.3) for the Super Twisting. Behavior of TAIPAN with these control laws is showed in figure (5) and figure (6) respectively. TAIPAN answer is asymptotical. The stabilization to the desired values is fester and precise than $\mathrm{PD}$ and SMC controllers, the improvement is more clear in $\theta$ and $q$ graphics.

\section{Conclusion and future work}

This paper describes PD, SMC and HOSMC control algorithms, which are evaluated in TAIPAN depth control. Experimental response of these controllers in the state space, depth $(z)$, pitch angle $(\theta)$, angular velocity $(q)$ and the output control $\left(\delta_{s}\right)$, are showed in the figure $3,4,5$ and 6 . The simulation results show that the performance for the
HOSMC is faster and precise than the other control laws, in all the states. This affirmation is more clear, when the states $\theta$ and $q$, are compared.

Future work includes the experimental results with HOSMC. The early results with this technique are promising to implemented in TAIPAN 2, a second generation of AUV currently developing at LIRMM.

\section{Acknowledgments}

The Authors are grateful to P. FRAISSE, from LIRMM, for helpful discussions.

\section{REFERENCES}

[1] G. Bartolini, A. Pisano and E. Usai, "Second order sliding mode control of container cranes", Automatica, no. 38,2002

[2] J. Bjorn, "The NDRE-AUV Flight Control system", IEEE journal of Oceanic Engineering, vol. 19, no. 4, October 1994.

[3] R. Cristi, A. Papoulias and A. J. Healey, "Adaptive sliding mode control of autonomous underwater vehicle in the dive plane", IEEE journal of Oceanic Engineering, vol. 15, no. 3, July 1990.

[4] A. I. Field, "Optimal control of an autonomous underwater vehicle", World Automatic Congress, Maui, Hawaii, June 11-16, 2000.

[5] T. I. Fossen, Guidance and control of oceans vehicles, John Wiley and Sons, 1994.

[6] A. J. Healey and D. Lienard, "Multivariable sliding mode control for autonomous diving and steering of unmanned underwater vehicles", IEEE journal of Oceanic Engineering, vol 18, no. 3, july 1993.

[7] H. Kawano and T. Ura, "Fast reinforcement learning algorithm for motion planning of non-holonomic underwater vehicle disturbance", Int. Conference on Intelligent Robots and systems IEEE/RSJ, Lausanne, Switzerland, October 2002.

[8] A. Levant, A. Pridor, R. Gitizadeh, I. Yaesh I. and J. Z Ben-Asher, "Aircraft pitch control via second-order sliding technique". AIAA Journal of Guidance, Control and Dynamics, vol. 23, no. 4, pp. 586-594, 2000.

[9] W. Perruquetti and J. P. Barbot, Sliding Modes control in Engineering. Marcel Dekker, Inc. 1999.

[10] T. Prestero, Verification of Six-Degree of freedom simulation model for the REMUS AUV. Master thesis, Massachusetts Institute of Technology, September 2001.

[11] L. Rodrigues, P. Tavares and M. Prado, "Sliding mode control of an AUV in the diving and steering planes", OCEANS MTS/IEEE. September 1996, Florida.

[12] J. Vaganay, B. Jouvencel, P. Lepinay and R. Zapata, "Taipan, an AUV for very shallow water applications", World Automatic Congress, Anchorage, 1998. 

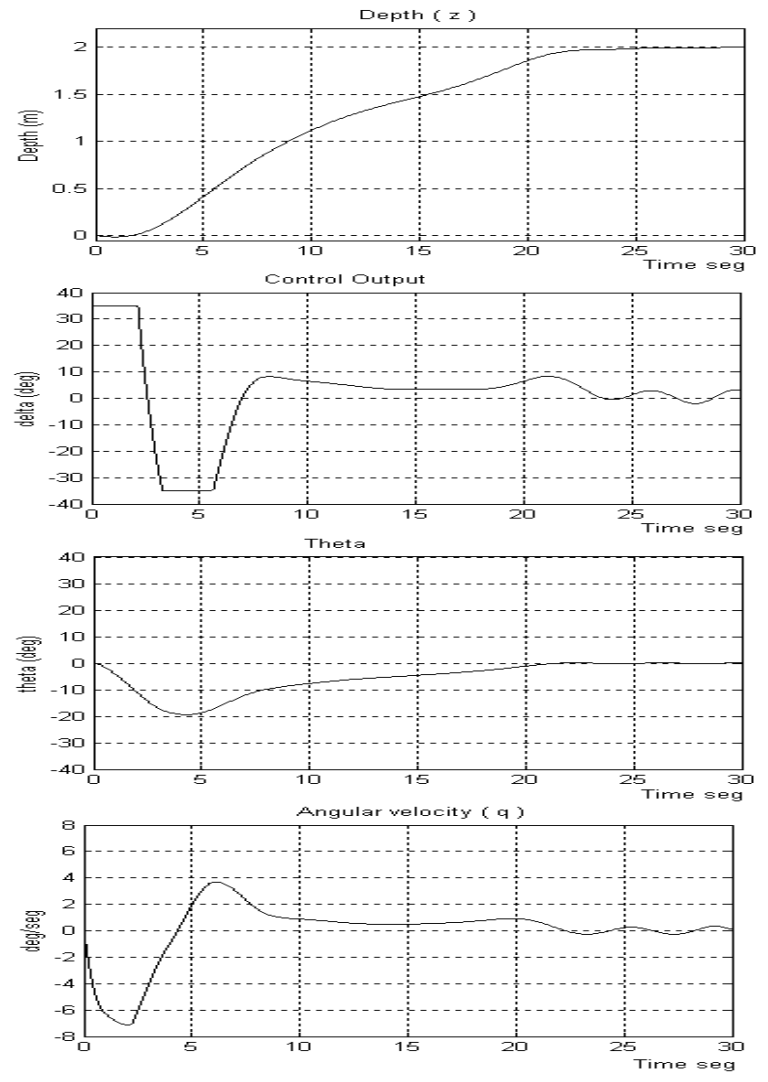

Fig. 3. TAIPAN Response to PD control law.
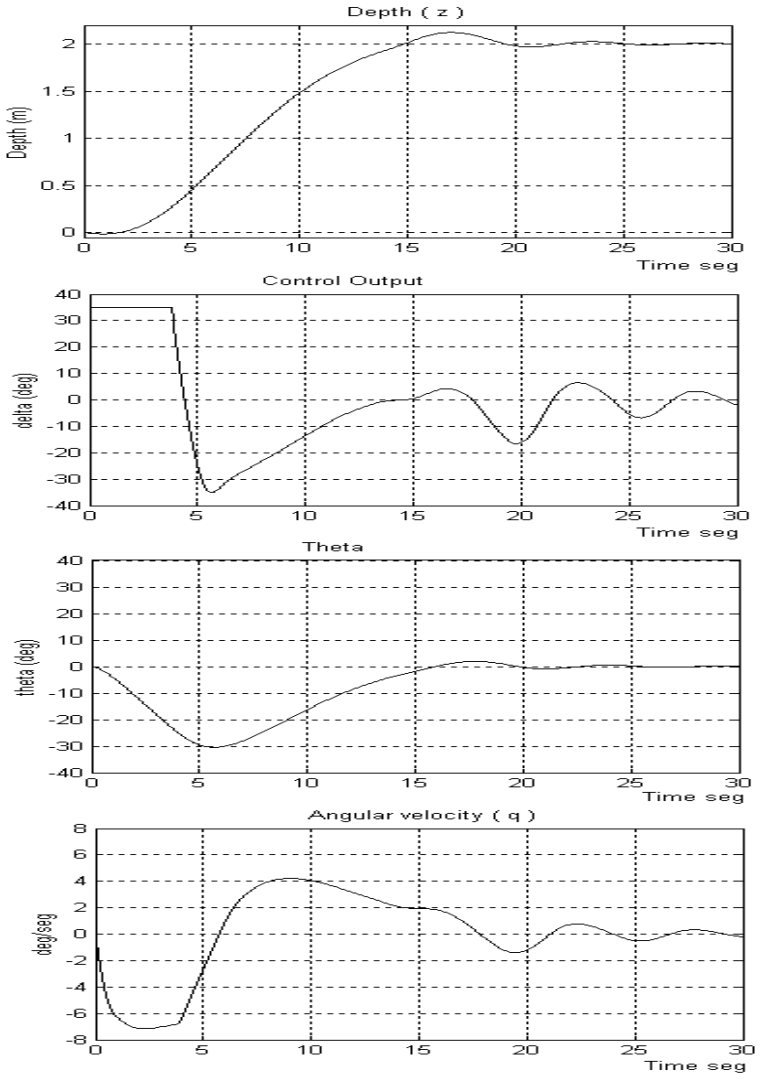

Fig. 4. TAIPAN Response to SMC control law.
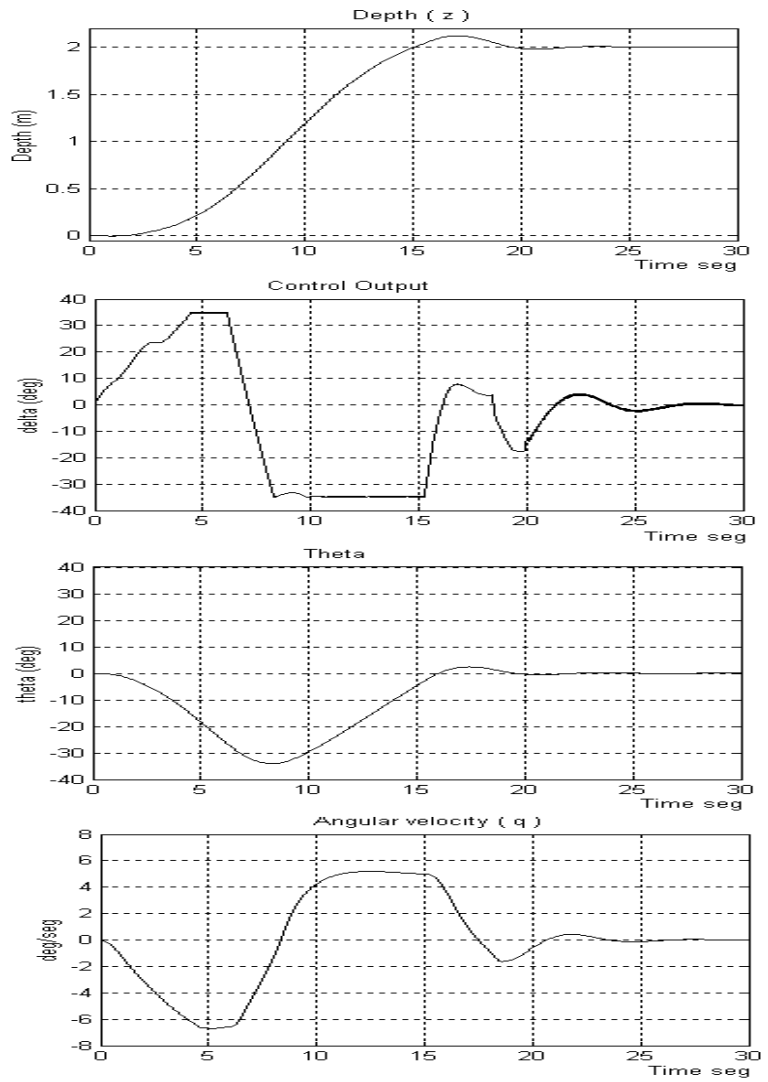

Fig. 5. TAIPAN Response to Twisting control law.
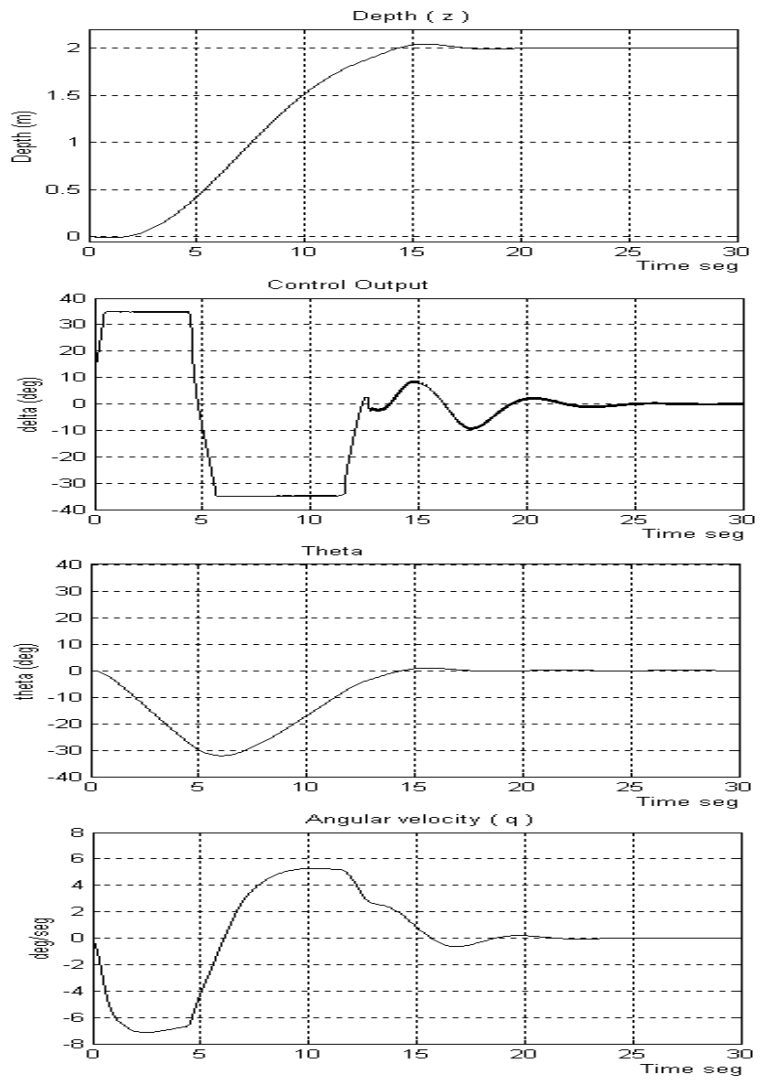

Fig. 6. TAIPAN Response to Super Twisting control law. 